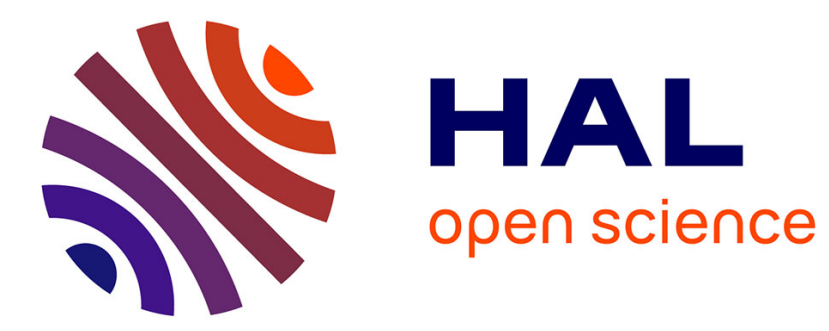

\title{
Rvb2/reptin physically associates with telomerase in budding yeast
}

Nathalie Grandin, Michel Charbonneau

\section{To cite this version:}

Nathalie Grandin, Michel Charbonneau. Rvb2/reptin physically associates with telomerase in budding yeast. FEBS Letters, 2011, 585, pp.3890-3897. 10.1016/j.febslet.2011.11.013 . hal-01154308

\section{HAL Id: hal-01154308 \\ https://hal-univ-tours.archives-ouvertes.fr/hal-01154308}

Submitted on 4 Nov 2015

HAL is a multi-disciplinary open access archive for the deposit and dissemination of scientific research documents, whether they are published or not. The documents may come from teaching and research institutions in France or abroad, or from public or private research centers.
L'archive ouverte pluridisciplinaire HAL, est destinée au dépôt et à la diffusion de documents scientifiques de niveau recherche, publiés ou non, émanant des établissements d'enseignement et de recherche français ou étrangers, des laboratoires publics ou privés. 


\section{Elsevier Editorial System(tm) for FEBS Letters \\ Manuscript Draft}

Manuscript Number: FEBSLETTERS-D-11-01347R1

Title: Rvb2/reptin physically associates with telomerase in budding yeast

Article Type: Research Letter

Keywords: telomerase; telomere length; Rvb2; budding yeast

Corresponding Author: Mr. Michel Charbonneau, Ph.D.

Corresponding Author's Institution: CNRS

First Author: Nathalie Grandin, Ph.D.

Order of Authors: Nathalie Grandin, Ph.D.; Michel Charbonneau, PhD; Michel Charbonneau, Ph.D.

Manuscript Region of Origin: FRANCE

Abstract: Telomerase is a reverse transcriptase that maintains linear telomeres at a constant length.

Here, we report that in the budding yeast Saccharomyces cerevisiae, Rvb2, a highly conserved member of the AAA+ family of ATPases, physically associates with telomerase/Est2 in vivo, both expressed from their endogenous promoter. Importantly, in genetic settings leading to a failure to recruit telomerase at telomeric ends, Rvb2 still associated with Est2. On the other hand, Rvb2 was present in immunoprecipitates of crosslinked telomeric chromatin even in the presumed absence of telomerase at the telomeres. Finally, we could also isolate RVB2 mutant alleles conferring slight, but stable, telomere shortening.

Suggested Reviewers:

Opposed Reviewers: 


\section{Rvb2/reptin physically associates with telomerase in budding yeast}

Nathalie Grandin ${ }^{1,2}$ and Michel Charbonneau ${ }^{1,2, *}$

${ }^{1}$ UMR CNRS 5239; Ecole Normale Supérieure de Lyon; IFR128 BioSciences Gerland;

69364 Lyon; France; ${ }^{2}$ UMR CNRS 6239; Université de Tours; 37200 Tours, France

Running head: Rvb2/reptin and telomerase

Key words: telomerase; telomere length; Rvb2; budding yeast

4,715 words

* Corresponding author's present address:

Michel CHARBONNEAU; UMR CNRS 6239; Université de Tours;

31, avenue Monge; Parc Grandmont; 37200 TOURS, FRANCE

Tel. (33) 247366089

FAX: (33) 247367042

e-mail: Michel.Charbonneau@univ-tours.fr

Nathalie.Grandin@univ-tours.fr 


\begin{abstract}
Telomerase is a reverse transcriptase that maintains linear telomeres at a constant length. Here, we report that in the budding yeast Saccharomyces cerevisiae, Rvb2, a highly conserved member of the AAAt family of ATPases, physically associates with telomerase/Est2 in vivo, both expressed from their endogenous promoter. Importantly, in genetic settings leading to a failure to recruit telomerase at telomeric ends, Rvb2 still associated with Est2. On the other hand, Rvb2 was present in immunoprecipitates of crosslinked telomeric chromatin even in the presumed absence of telomerase at the telomeres. Finally, we could also isolate RVB2 mutant alleles conferring slight, but stable, telomere shortening.
\end{abstract}


S. cerevisiae telomerase (Est2) and Rvb1 and Rvb2 physically associate. Rvb2 was present in telomeric chromatin following immunoprecipitation under cross link conditions. Rvb2 still associated with Est2 in cells in which telomeras/Est2 could not be recruited at telomeric ends. $r v b 2$ mutant alleles conferring slight, but stable, telomere shortening could be isolated. 


\section{Introduction}

Telomerase is a reverse transcriptase with a built-in RNA template that is essential for maintaining functional telomeres at a constant length in most eukaryotic organisms. Maintenance of a constant telomere length is essential to protect the chromosomes from undergoing degradation $(1,2)$. In mammals this protection is provided mainly by the so-called shelterin complex, a complex of six telomeric proteins (TRF1, TRF2, POT1, TIN2, TPP1 and RAP1) that prevent inappropriate fusion between telomeres as well as unwanted telomeric recombination (3). A rather similar complex exists in the fission yeast Schizosaccharomyces pombe (4), while in the budding yeast Saccharomyces cerevisiae, a somewhat simpler protection complex, consisting mainly of the $\mathrm{Cdc} 13, \operatorname{Stn} 1$ and Ten1 proteins (sometimes referred to as the CST complex) is present (5). However, very recently the orthologs of $S$. cerevisiae CST were found in humans as well as in S. pombe and Arabidopsis thaliana (6). A number of accessory proteins are required for the assembly of telomerase (7). Subsequently, distinct proteins are required for the loading of telomerase onto the telomeres as well as for its activation. It is not currently known in detail how telomerase, which is present at the telomeres even when it is not performing its telomere elongation function, can attain its activation state.

Very recently, pontin and reptin, two highly conserved members of the AAA+ family of ATPases (putative DNA helicases), initially named RUVBL1 and RUVBL2 after the bacterial RuvB-Holliday-junction migrating helicase, were found to associate with both subunits of human telomerase (8). In both S. cerevisiae and humans, Rvb1/pontin and Rvb2/reptin are parts of several complexes involved in chromatin modification and also play major roles in the regulation of transcription (9-12). In S. cerevisiae, there has been so far no interaction, physical, genetic or other between Rvb1 or Rvb2 and telomerase reported in the literature. We report here the existence in $S$. cerevisiae of a physical association between $\mathrm{Rvb2}$ and telomerase and the isolation of $R V B 2$ mutants with shortened telomeres.

\section{Materials and methods}

\subsection{Yeast strains, plasmids and inducible overexpression by galactose}

Yeast strains used in this study, derivatives of BF264-15Daub (ade1 his2 leu2-3,112 trp1-1a ura3Dns), were cultured as described previously (13). All strains were made isogenic by back crossing at least five times against our genetic background. Strain origins, prior to back crossing, were as follows. The rvb2::KanMX4/RVB2, est2::KanMX4/EST2, tel1::KanMX4 and 
$y k u 70:: K a n M X 4$ strains were from Euroscarf (Frankfurt, Germany). The $t l c 1:: T R P 1$ strain was from the Gottschling laboratory.

All constructs were made by using Polymerase Chain Reaction (PCR) to adapt the relevant restriction sites to the sequence of the genes and details of the constructs can be available upon request. Induction of genes placed under the control of the GAL1-10 promoter, in a high-copy vector (episomal, $2 \mu$ ), was done by transferring cells growing in glucosecontaining liquid medium into galactose-containing liquid medium after several washes by centrifugation and allowing overexpression for $2 \mathrm{hr}$ at $29^{\circ} \mathrm{C}$. In experiments involving expression of protein parts under GAL1-10 promoter control a supplementary methionine residue was added in front of the truncated sequence (if starting from other places than the natural initiating ATG) to initiate efficient translation.

\subsection{Immunoprecipitation and immunoblotting}

Mouse monoclonal anti-HA raw ascites fluid 16B12 (BabCO) and mouse monoclonal antiHA 12CA5 antibody (Roche Diagnostics) were used for immunoprecipitation and imunoblotting, respectively. Myc-tagged proteins were visualized after immunoprecipitation and Western blotting with monoclonal anti-Myc antibody 9E10 (Roche Diagnostics).

Techniques for cell extract preparation, immunoprecipitation and immunoblotting (analyzed using an Odyssey Imager), as well as for block and release experiments, have been described previously $(13,14)$.

To perform immunoprecipitation-Western blotting experiments following conditions of protein-DNA crosslink, cell cultures were treated with 1\% formaldehyde for 15-30 min at the selected temperature for growth. Cell extracts were then prepared as usual but, here, chromatin was shredded by sonication. The rest of the protocol for immunoprecipitation and Western blotting was as usual.

\subsection{Generation of RVB2 mutant alleles and measurement of telomere lengths on teloblots}

To mutagenize $R V B 2$, the 1413 base pair (bp)-long $R V B 2$ ORF plus $\sim 310$ bp upstream of the ATG and $\sim 300$ bp downstream of the stop codon was amplified by error-prone PCR under the following conditions. The concentration of dNTPs was either kept as in standard conditions (200 $\mu \mathrm{M}$ each) or one of the four dNTP concentration changed to $0.5-1.0 \mathrm{mM}$, those of the other three being kept at $200 \mu \mathrm{M}$, and, in both cases, the concentration of $\mathrm{MgCl}_{2}$ was changed from 1.5 to $3.0 \mathrm{mM}$. Standard Taq polymerase and PCR buffer (Promega) were used. Following a 30-cycle amplification, the mutated fragments were transformed into an 
$r v b 2:: K a n M X 4$ strain carrying wild-type $R V B 2$ on a $C E N-U R A 3$ plasmid, together with a centromeric, TRP1-based (YCplac22), plasmid carrying RVB2 ORF plus the same flanking sequences and made linear by digestion with $X b a \mathrm{I}$ and $N c o \mathrm{I}$, endogenous sites located $\sim 90$ base pairs post-initiating ATG and $\sim 115$ base pairs downstream of the stop codon, respectively. After counter-selection on 5-FOA medium, transformants having extruded wildtype $R V B 2$ were picked out randomly from the plates, propagated for $\sim 20$ days at $24^{\circ} \mathrm{C}$ to attain telomere length equilibrium.

To analyze telomere length by Southern blotting, genomic DNAs were prepared, as described previously (14). Following digestion of genomic DNA with XhoI, to cut within the $\mathrm{Y}^{\prime}$ regions of chromosomes, telomere tracts of wild-type cells appear as a broad band of 1.1-1.3 kb which represents the average length of most chromosomes. Results were analyzed using an FLA-5100 Fuji phosphoimager and the ImageGauge software.

\section{Results and discussion}

\subsection{Rvb2 associates with telomerase}

The finding that human pontin and reptin (homologues to Rvb1 and Rvb2, respectively), two closely related members of the AAA+ family of ATPases, physically associate with telomerase (8) prompted us to examine whether similar physical interactions existed in budding yeast. In asynchronous cells, a small amount of $\mathrm{HA}_{2}-\mathrm{Rvb} 2$ could be detected following immunoprecipitation of $\mathrm{Myc}_{18}$-Est2 using anti-Myc monoclonal antibody, both expressed from their respective genomic locus under native promoter control (Figure 1A). Since Rvb2 is much more abundant within the cell than Est2 (15), we verified that the amount of $\mathrm{HA}_{2}$-Rvb2 immunoprecipitated by $\mathrm{Myc}_{18}$-Est2 was reproducibly higher than the background level of $\mathrm{HA}_{2}-\mathrm{Rvb} 2$ (Figure 1A and data not shown). The in vivo interaction between $\mathrm{HA}_{2}-\mathrm{Rvb2}$ and $\mathrm{Myc}_{18}$-Est2 was observed throughout the cell cycle, in cells previously synchronized in G1 phase with alpha-factor (Figure 1B). This indicates that in budding yeast, unlike in humans (8), the Est2-Rvb2 interaction does not appear to peak in $\mathrm{S}$ phase. In the converse experiment, we could not detect $\mathrm{Myc}_{18}$-Est2 in the $\mathrm{HA}_{2}-\mathrm{Rvb2}$ immunoprecipitate (data not shown). Since Rvb2 has been found in physical association with several other proteins in distinct complexes (12), this tends to support the idea that a small fraction only of Rvb2 is in complex with Est2. This conclusion should however be toned down by the fact that $\mathrm{Myc}_{18}$-Est2 being much less abundant than $\mathrm{HA}_{2}-\mathrm{Rvb} 2$, the amount of $\mathrm{Myc}_{18}$-Est2 immunoprecipitated by $\mathrm{HA}_{2}$-Rvb2 might be too small to be detected. 
The in vivo interaction between Rvb2 and Est2 was very reproducible but small, and we needed confirmation. Therefore, we next examined Rvb2-Est2 physical interactions under conditions of increased expression by using a GAL1-10 promoter-controlled inducible system. By controlling the duration of induction of the promoter in galactose-based liquid culture medium, one can limit potentially deleterious effects of heavy overexpression. Such a system is currently used in genome-wide analyses because it presents the advantage compared to other systems of assessing in vivo interactions between proteins in their native configuration. Upon induction of the GAL1-10 promoter for $2 \mathrm{hr}$ at $29^{\circ} \mathrm{C}$, the amount of Rvb2-HA 2 coprecipitating with either one of two Est2-Myc 3 parts, one of which, Est2 ${ }^{420-740}-\mathrm{Myc}_{3}$, comprising the entire catalytic domain, was higher than the background level of Rvb2- $\mathrm{HA}_{2}$ in the strain expressing the GAL- $R V B 2-\mathrm{HA}_{2}$ construct alone (Figure 1C).

Repeated attempts to obtain strains expressing an epitope-tagged $R V B 1$ construct from its genomic locus remained unsuccessful for unknown reasons. We therefore attempted to detect a possible physical interaction between Rvb1-HA 2 and one of the two Est2-Myc 3 parts using the GAL1-10 overexpression system described above. As shown in Figure 1D, like with Rvb2-HA 2 , physical association between Rvb1-HA 2 and Est2 ${ }^{420-740}-\mathrm{Myc}_{3}$, corresponding to the catalytic domain, could be detected.

Recently, Ies3, a member of the INO80 complex, was found to undergo a weak physical interaction with Est1 (16). Est1 is a single-stranded telomeric DNA-binding protein that loads telomerase onto the telomeric ends $(17,18)$. Est1 also binds Est2, through bridging with TLC1, the RNA template subunit of telomerase (19). Since Rvb2 is part of the INO80 complex (12), we asked whether physical interactions between Est1 and Rvb2 could be detected. Est1-Myc ${ }_{3}$ failed to bind Rvb2-HA $($ Figure 1E). This demonstrated that, contrary to Est2, Est1 has no apparent affinity with Rvb2, at least when both are overproduced.

\subsection{Rvb2 is present in crosslinked telomeric chromatin}

We next asked whether Rvb2 might be part of telomeric chromatin. Chemical crosslinking with formaldehyde and related reagents has been widely used to immobilize protein-DNA contacts for ChIP as well as to fix sub-cellular structures for microscopy (20-22). We decided to apply a crosslink protocol prior to immunoprecipitation using a strain expressing a CDC13$\mathrm{Myc}_{13}$ construct from genomic locus under the control of its native promoter (23). Cdc13 is the major single-stranded telomeric DNA-binding protein in budding yeast and has an essential function both in telomere end protection and telomerase recruitment $(5,24)$. We reasoned that based on ChIP experiments on telomeric proteins in which telomeric DNA is 
detected by PCR in the immunoprecipitate, we might be able to detect $\mathrm{HA}_{2}-\mathrm{Rvb2}$ in the telomeric chromatin immunoprecipitated by $\mathrm{Cdc} 13-\mathrm{Myc}_{13}$. Only under conditions in which the experiment had been performed under crosslink conditions was the association between Cdc13-Myc ${ }_{13}$-immunoprecipitated telomeric chromatin and $\mathrm{HA}_{2}-\mathrm{Rvb} 2$ clearly visible (Figure 2A). Bmh1 was used as a control for these experiments because it has a very high intracellular concentration (in fact, Bmh1 is far more abundant than Rvb2, 158,000 and $~ 3,030$ molecules/cell, respectively, while a rare telomeric protein such as $\mathrm{Cdc} 13$ is present at only 320 molecules/cell; ref. 15) and, therefore, cannot be suspected of being undetected in the anti-Myc immunoprecipitate because of too low an intracellular level. We could not detect any physical association between $\mathrm{Cdc} 13-\mathrm{Myc}_{13}$ and $\mathrm{HA}_{2}-\mathrm{Bmh} 1$ whether under crosslink or native conditions, while the $\mathrm{HA}_{2}-\mathrm{Rvb} 2$ sample scored positive within the same experiment (Figure 2A). The presence of HA-Rvb2 in the crosslinked immunoprecipitate appeared to occur also in the absence of a Myc tag on Cdc13, albeit at a reduced level (Figure 2A). Although this cannot be ignored, it should be noted that this background level of $\mathrm{HA}_{2}-\mathrm{Rvb2}$ was constantly observed throughout the course of the present study, whether under native or crosslink immunoprecipitation experiments (Figure 1A, 2A). However, the fact is that the increased $\mathrm{HA}_{2}-\mathrm{Rvb} 2$ signal observed following immunoprecipitating $\mathrm{Cdc} 13-\mathrm{Myc}_{13}$ necessarily corresponded to a specific signal. This phenomenon most probably results from the intrinsically high levels of intracellular Rvb2 as it was also observed with the highly abundant Bmh1 protein. However, most importantly, with $\mathrm{HA}_{2}-\mathrm{Bmh} 1$ the background level did not increase upon $\mathrm{Cdc} 13-\mathrm{Myc}_{13}$ immunoprecipitation, thus strongly suggesting an absence of association between $\mathrm{HA}_{2}-\mathrm{Bmh} 1$ and $\mathrm{Cdc} 13-\mathrm{Myc}_{13}$-bound chromatin, contrary to the case with $\mathrm{HA}_{2}-\mathrm{Rvb} 2$ (Figure 2A).

The interactions between Rvb2 and telomeric DNA did not change during a synchronous cell cycle obtained after alpha-factor pheromone block and release (Figure 2B).

$\mathrm{HA}_{2}-\mathrm{Rvb} 2$ was still present in Cdc13-Myc ${ }_{13}$-immunoprecipitated telomeric chromatin in the absence of functional telomerase, namely in strains deleted for the TLC1 RNA subunit of telomerase or in the est1 null mutant (Figure 2C). Previous experiments have demonstrated that in the absence of $T L C 1$, Est1 and Est2 no longer physically interact (19). Therefore, the present experiments strongly suggest that Rvb2 is present at the telomeres even in the absence of telomere-recruited Est2. However, further experiments using a different approach will be necessary to definitively establish this potentially important finding. 
3.3. The Rvb2-Est2 interaction takes place even when telomerase is not present at the telomeres

The Rvb2-Est2 interaction described above might potentially take place at the telomeres but nothing excluded the possibility that Rvb2 could associate with Est2 at locations other than the telomeres. To explore this possibility, we took advantage of previously reported in vivo findings showing that both the YKU complex, during the G1 phase and early S phase (25), and Est1, from the end of $\mathrm{S}$ phase to the end of mitosis $(17,26,27)$, are required for telomerase loading at the telomeres and, importantly, that inhibiting these two pathways totally prevent the presence of telomerase on the telomeres (26). As expected, combining the est 1 null and $y k u 70$ null mutations led to greatly accelerated senescence, leaving too little time to grow enough cells to conduct the experiments prior to senescence (data not shown). Therefore, the experiments were performed on est1 null yku70 null cells undergoing postsenescence telomeric recombination. Not only did this not impinge on the desired experimental design but, in addition, confirmed that all telomerase was completely off the telomeres, as cells incapable of loading telomerase at their telomeres undergo telomeric senescence followed, in a small minority of them, by post-senescence survival by telomeric recombination $(28,29)$. Interestingly, in an est 1 null yku70 null double mutant (Figure 3A) as well as in the est 1 null mutant (Figure 3B), $\mathrm{HA}_{2}-\mathrm{Rvb} 2$ was found to still physically associate with $\mathrm{Myc}_{18}$-Est2 to levels similar to that in the wild type. We note that the amount of $\mathrm{Myc}_{18^{-}}$ Est2 was larger in the est 1 yku70 double mutant than in the wild-type but additional experiments would be required to conclude on this point.

It should be noted that in the est1 null mutant, TLC1 RNA was found to mostly accumulate in the cytoplasm (30), thus making unlikely the presence of an abundant and functional Est2-TLC1 complex at the telomeres in that mutant. On the other hand, deletion of EST1 did not prevent the formation of an Est2-TLC1 complex (31), which would therefore be cytoplasmic in that mutant (30). Altogether, this indicates the physical interaction between Rvb2 and Est2 detected in the present study presumably takes place in the cytoplasm (or possibly but unlikely in the nucleus but off the telomeres) during maturation and/or assembly of telomerase (7).

\section{4. rvb2 mutants with shortened telomeres}

Since Rvb2 and telomerase physically associate, we next set out to identify possible $r v b 2$ mutants deregulated in telomere length control. Two hundred colonies resulting from the transformation of an $r v b 2$ null strain with PCR-mutagenized RVB2 (see Materials and 
methods) were picked out randomly from the plates and individually propagated for $\sim 20$ days at $24^{\circ} \mathrm{C}$ so that they could attain telomere length equilibrium. Under these conditions, we could select, by Southern blot analysis of the terminal restriction fragments, five $r v b 2$ mutants with altered telomere length, which all exhibited slight telomere shortening (Figure 4A). None of these mutants exhibited signs of cellular senescence, a phenomenon that takes place when telomeres erode beyond a critical threshold (data not shown). Upon propagation during additional cell divisions, the decreased telomere length was found to be stable in all five selected mutants (Figure 4B). Therefore, telomere shortening in the isolated $r v b 2$ mutants is stabilized at a shorter than wild type value, just like in the deletion mutants of the YKU or MRX complexes or of TEL1 (32-34), although the effects of these later mutations are more dramatic (Figure 4B). All mutations (Rvb2-2 and Rvb2-3: A50T, T388A; Rvb2-24 and Rvb2-26: T99A; Rvb2-94: T262A, N310D, I370M, all in the rvb2::Kan-MX4 YCplac22-pro$r v b 2$-tail genetic context) were found to localize to residues that have been conserved between the budding yeast and human proteins, and, in addition, were outside of the Walker A and B ATPase motifs, which serve to bind ATP (Figure 4C). Incidentally, both budding yeast Rvb1 and Rvb2 have been recently shown to exhibit ATPase activity, as well as ATP-dependent DNA helicase activity in vitro with increased activities when the two proteins were in complex (35).

The relatively modest telomere shortening of the isolated $r v b 2$ mutants have so far prevented us from uncovering interesting genetic interactions with other mutations previously implicated in telomere maintenance ( $\mathrm{NG}, \mathrm{MC}$, unpublished data).

\subsection{Conclusions}

The major finding of the present study is to have uncovered a weak, albeit very reproducible, in vivo physical interaction between Rvb2/reptin and the catalytic subunit of budding yeast telomerase, Est2. Given the status of budding yeast $S$. cerevisiae and humans as two of the major biological model systems to study telomeres, this data is of major importance in light of the existence of a similar interaction between human telomerase and reptin (8). Altogether, the present data suggest that Rvb2 may specifically associate with Cdc13-Myc ${ }_{13}$-bound telomeric chromatin in telomerase-independent transactions. We speculate that the Rvb1/2 helicases (35) may assist in changing the conformation of telomeric ends so as to optimize telomerase recruitment, perhaps also taking advantage of the potentially important Rvb2-Est2 physical association shown here. 


\section{Acknowledgements}

We are very grateful to Craig Peterson for the gift of the Cdc13-Myc 13 plasmid. This work was supported by grants from the "LIGUE contre le Cancer, Comité inter-régional RhôneAlpes Auvergne". 


\section{References}

1. Grandin, N and Charbonneau, M. (2008). Protection against chromosome degradation at the telomeres. Biochimie 90, 41-59.

2. O’Sullivan, R.J. and Karlseder, J. (2010) Telomeres: protecting chromosomes against genome instability. Nat. Rev. Mol. Cell. Biol. 11, 171-181.

3. Palm, W. and de Lange, T. (2008) How shelterin protects mammalian telomeres. Annu. Rev. Genet. 42, 301-334.

4. Moser, B.A. and Nakamura, T.M. (2009) Protection and replication of telomeres in fission yeast. Biochem. Cell. Biol. 87, 747-758.

5. Shore, D. and Bianchi, A. (2009) Telomere length regulation: coupling DNA end processing to feedback regulation of telomerase. EMBO J. 28, 2309-2322.

6. Giraud-Panis, M.J., Teixeira, M.T., Géli, V. and Gilson, E. (2010) CST meets shelterin to keep telomeres in check. Mol. Cell 39, 665-676.

7. Gallardo, F. and Chartrand, P. (2008) Telomerase biogenesis - The long road before getting to the end. RNA Biol. 5, 212-215.

8. Venteicher, A.S., Meng, Z., Mason, P.J., Veenstra, T.D. and Artandi, S.E. (2008) Identification of ATPases Pontin and Reptin as telomerase components essential for holoenzyme assembly. Cell 132, 945-957.

9. Lim, C.R., Kimata, Y., Ohdate, H., Kokubo, T., Kikuchi, N., et al. (2000) The Saccharomyces cerevisiae RuvB-like protein, Tih2p, is required for cell cycle progression and RNA polymerase II-directed transcription. J. Biol. Chem. 275, 22409-22417.

10. Gallant, P. (2007) Control of transcription by Pontin and Reptin. Trends Cell Biol. 17, 187-192. 
11. Shevchenko, A., Roguev, A., Schaft, D., Buchanan, L., Haberman, B., et al. (2008) Chromatin central: towards the comparative proteome by accurate mapping of the yeast proteomic environment. Genome Biol. 9, R167.

12. Jha, S. and Dutta, A. (2009) RVB1/RVB2: running rings around molecular biology. Mol. Cell 34, 521-533.

13. Grandin, N. and Reed, S.I. (1993) Differential function and expression of Saccharomyces cerevisiae B-type cyclins in mitosis and meiosis. Mol. Cell. Biol. 13, 2113-2125.

14. Grandin, N., Reed, S.I. and Charbonneau, M. (1997) Stn1, a new Saccharomyces cerevisiae protein, is implicated in telomere size regulation in association with $\mathrm{Cdc} 13$. Genes Dev. 11, 512-527.

15. Ghaemmaghami, S., Huh, W.K., Bower, K., Howson, R.W., Belle, A., et al. (2003) Global analysis of protein expression in yeast. Nature $425,737-741$.

16. Yu, E.Y., Steinberg-Neifach, O., Dandjinou, A.T., Kang, F., Morrison, A.J., et al. (2007) Regulation of telomere structure and functions by subunits of the INO80 chromatin remodeling complex. Mol. Cell. Biol. 27, 5639-5649.

17. Evans, S.K. and Lundblad, V. (1999) Est1 and Cdc13 as comediators of telomerase access. Science 286, 117-120.

18. Lendvay, T.S., Morris, D.K., Sah, J., Balasubramanian, B. and Lundblad, V. (1996) Senescence mutants of Saccharomyces cerevisiae with a defect in telomere replication identify three additional EST genes. Genetics 144, 1399-1412.

19. Evans, S.K. and Lundblad, V. (2002) The Est1 subunit of Saccharomyces cerevisiae telomerase makes multiple contributions to telomere length maintenance. Genetics 162, 1101 1115 . 
20. Solomon, M. and Varshavsky, A. (1985) Formaldehyde-mediated DNA-protein crosslinking: a probe for in vivo chromatin structures. Proc. Natl. Acad. Sci. USA 82, 64706474.

21. Gilmour, D.S., Rougvie, A.E. and Lis, J.T. (1991) Protein-DNA cross-linking as a means to determine the distribution of proteins on DNA in vivo. Methods Cell. Biol. 35, 369-381.

22. Orlando, V., Strutt, H. and Paro, R. (1997) Analysis of chromatin structure by in vivo formaldehyde cross-linking. Methods 11, 205-214.

23. Oza, P., Jaspersen, S.L., Miele, A., Dekker, J. and Peterson, C.L. (2009) Mechanisms that regulate localization of a DNA double-strand break to the nuclear periphery. Genes Dev. 23, 912-927.

24. Garvik, B., Carson, M. and Hartwell, L. (1995) Single-stranded DNA arising at telomeres in $c d c 13$ mutants may constitute a specific signal for the RAD9 checkpoint. Mol. Cell. Biol. $15,6128-6138$.

25. Fisher, T., Taggart, A. and Zakian, V. (2004) Cell cycle-dependent regulation of yeast telomerase by Ku. Nat. Struct. Mol. Biol. 11, 1198-1205.

26. Chan, A., Boulé, J.B. and Zakian, V.A. (2008) Two pathways recruit telomerase to Saccharomyces cerevisiae telomeres. PLoS Genet. 4, e1000236.

27. Taggart, A.K.P., Teng, S. and Zakian, V.A. (2002) Estlp as a cell cycle-regulated activator of telomere-bound telomerase, Science 297, 1023-1026.

28. Lundblad, V. and Szostak, J.W. (1989) A mutant with a defect in telomere elongation leads to senescence in yeast. Cell 57, 633-643.

29. Lundblad, V. and Blackburn, E.H. (1993) An alternative pathway for yeast telomere maintenance rescues est ${ }^{-}{ }^{-}$senescence. Cell 73, 347-360. 
30. Gallardo, F., Olivier, C., Dandjinou, A.T., Wellinger, R.J. and Chartrand, P. (2008) TLC1 RNA nucleo-cytoplasmic trafficking links telomerase biogenesis to its recruitment to telomeres. EMBO J. 27, 748-757.

31. Hughes, T.R., Evans, S.K., Weilbaecher, R.G. and Lundblad, V. (2000) The Est3 protein is a subunit of yeast telomerase. Curr. Biol. 10, 809-812.

32. Lustig, A.J. and Petes, T.D. (1986) Identification of yeast mutants with altered telomere structure. Proc. Natl. Acad. Sci. USA 83, 1398-1402.

33. Kironmai, K.M. and Muniyappa, K. (1997) Alteration of telomeric sequences and senescence caused by mutations in RAD50 of Saccharomyces cerevisiae. Genes Cells 2, 443455.

34. Boulton, S.J. and Jackson, S.P. (1998) Components of the Ku-dependent nonhomologous end-joining pathways are involved in telomeric length maintenance and telomeric silencing. EMBO J. 17, 1819-1828.

35. Gribun, A., Cheung, K.L.Y., Huen, J., Ortega, J. and Houry, W.A. (2008) Yeast Rvb1 and Rvb2 are ATP-dependent DNA helicases that form a heterohexameric complex. J. Mol. Biol. $376,1320-1333$.

36. Lingner, J., Hughes, T.R., Shevchenko, A., Mann, M., Lundblad, V. and Cech, T.R. (1997) Reverse transcriptase motifs in the catalytic subunit of telomerase. Science 276, 561567. 


\section{Legends of figures}

Figure 1: Rvb2 physically associates with Est2/telomerase, but not with Est1. (A) Cell extracts from asynchronous wild-type cells harboring either $\mathrm{HA}_{2}-R V B 2$ or $\mathrm{Myc}_{18}-E S T 2$ constructs or both. After separation by gel electrophoresis, the immunoprecipitates (IP) were revealed by the corresponding monoclonal antibodies as indicated (West.). (B) Cells from a bar1 mutant expressing both $\mathrm{HA}_{2}-R V B 2$ and $\mathrm{Myc}_{18}-E S T 2$ (from their respective genomic locus) were synchronized in G1 with alpha-factor, released from the block point by washing out the pheromone and analyzed at the indicated positions of the cell cycle (assessed in parallel by FACS analysis) for the existence of a physical interaction between the two proteins. First lane is the control using asynchronous cells from a strain expressing $\mathrm{HA}_{2}-R V B 2$ alone. (C) Immunoprecipitation-Western blotting experiments using strains overexpressing either full length $R V B 2-\mathrm{HA}_{2}$ or parts of EST2-Myc 3 (amino acids numbers indicated; Est $2^{420-}$ ${ }^{740}-\mathrm{Myc}_{3}$ comprises the entire catalytic domain; ref. 36) ORFs, or both. (D) Rvb1-HA physically associates with the fragment of the catalytic subunit of telomerase, Est $2^{420-740}$ $\mathrm{Myc}_{3}$, corresponding to its catalytic domain. (E) Est1-Myc $\mathrm{M}_{3}$ does not physically associate with

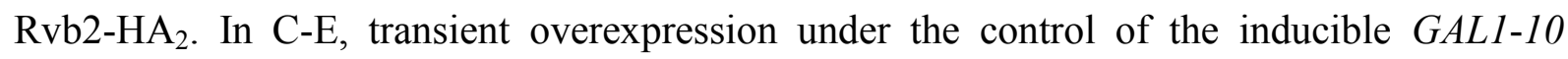
promoter on a high-copy vector (episomal, $2 \mu$ ) was induced in the liquid cultures for $2 \mathrm{hr}$ at $29^{\circ} \mathrm{C}$.

Figure 2: $\mathrm{HA}_{2}-R V B 2$ is present in $\mathrm{Cdc} 13-\mathrm{Myc}_{13}$-immunoprecipitated telomeric chromatin. (A) Strains expressing either $\mathrm{HA}_{2}-R V B 2$ or $\mathrm{HA}_{2}-B M H 1$ (native promoter, chromosomal locus) were processed as described above (no crosslink, top panels) or under crosslink conditions with formaldehyde (bottom panels). Under crosslink conditions $\mathrm{HA}_{2}-\mathrm{Rvb} 2$ but not $\mathrm{HA}_{2}$-Bmh1 could be detected in $\mathrm{Cdc} 13-\mathrm{Myc}_{13}$ immunoprecipitate. It is important to note that the $\mathrm{HA}_{2}$-Rvb2 IP-West., $\mathrm{HA}_{2}$-Bmh1 IP-West and Cdc13-Myc 13 IP-West. (bottom three rows in each panel) were performed on the same small volume (1/50 of total extract) in order to avoid ending up with saturating amounts of Rvb2 and Bmh1, while, on the opposite, Cdc13 which is far less abundant than Rvb2 and Bmh1 yielded a much weaker signal (see main text). In all crosslink experiments performed, the amount of IP-West. signal for a given protein was always much lower than in the absence of formaldehyde (compare bottom three rows of top panel with bottom three rows of bottom panel). We believe that either the extraction of the proteins by IP is less efficient due to the presence of formaldehyde and/or that a significant number of cells die during the crosslink (prior to the IP). (B) Rvb2 associates with telomeric 
chromatin throughout the cell cycle. Cell cycle progression was followed by scoring under the microscope for the principal cell cycle stages (in duplicates fixed in parallel) schematically represented here. Unbudded cells were in $\mathrm{G} 1$ or G1/S; cells with small buds were initiating S phase and cells with large buds were at G2/M. (C) Same experiments as above, but performed in the $t l c 1$ (TLC1 codes for the RNA subunit of telomerase) null or est1 null background, as indicated.

Figure 3: Endogenous Rvb2 and Est2 still physically associate when telomerase access to the telomeres is restricted or even totally prevented. (A) Immunoprecipitation-Western blotting experiments (IP-West.) on $\mathrm{HA}_{2}-R V B 2$ and $\mathrm{Myc}_{18}-E S T 2$ in an est1 yku70 null background, in which telomerase access to the telomeres is completely eliminated, under native IP-West. conditions. It is important to note that both the $\mathrm{HA}_{2}$-Rvb2 IP-West. and $\mathrm{Myc}_{18}$-Est2 IP-West. (bottom two rows) were performed on the same small volume (1/50 of total extract) in order to avoid ending up with saturating amounts of Rvb2, while, on the opposite, Est 2 was barely detected in such a small volume of cell extract because it is far less abundant than Rvb2 (see main text). (B) Same as above in a strain harboring an est 1 null mutation ( $3^{\text {rd }}$ lane) compared with the wild type background $\left(2^{\text {nd }}\right.$ lane $)$. The $1^{\text {st }}$ lane is a control with no $\mathrm{Myc}_{18}-E S T 2$ expression to assess for $\mathrm{HA}_{2}-\mathrm{Rvb} 2$ background.

Figure 4: Mutations in RVB2 confer slight telomere shortening. (A) Genomic DNAs were prepared from individual strains containing PCR-mutagenized $R V B 2$ and each lane corresponds to a single strain. Southern blotting with a telomeric $\mathrm{P}^{32}$-labeled probe revealed the size of the bulk of terminal $\mathrm{TG}_{1-3}$ telomere tracts, corresponding to the DNA smear migrating at around 1.2-1.3 $\mathrm{kb}$. The dashed horizontal line, representing the mean wild-type (wt) length, allowed us to obtain a better appreciation of the variations in telomere length from the wild-type value in the five indicated mutants. (B) Plasmids harboring mutant $r v b 2$ alleles were recovered from the selected original mutants, presented above, in A, transformed back into the original strains and the resulting transformants, illustrated here, further propagated in culture for 22 days at $24^{\circ} \mathrm{C}$ in order to make sure that the initially recorded values were maintained constant with time. Shortened telomeres of $y k u 70$ null and $x r s 2$ null strains (Xrs2 is part of the Mre11-Rad50-Xrs2 complex) are shown for comparison. (C) Positions of the mutations in Rvb2 sequence shown aligned with its human homologue. The positions of Walker A and B motifs are shown. 
Figure 1

A

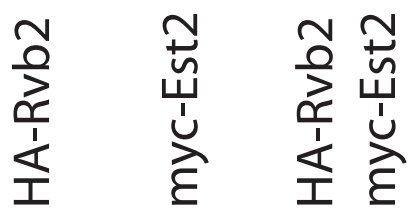

HA-Rvb2

IP myc-Est2, West. HA-Rvb2

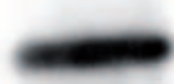

HA-Rvb2

IP HA-Rvb2, West. HA-Rvb2

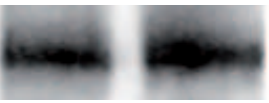

myc-Est2

IP myc-Est2, West. myc-Est2

B

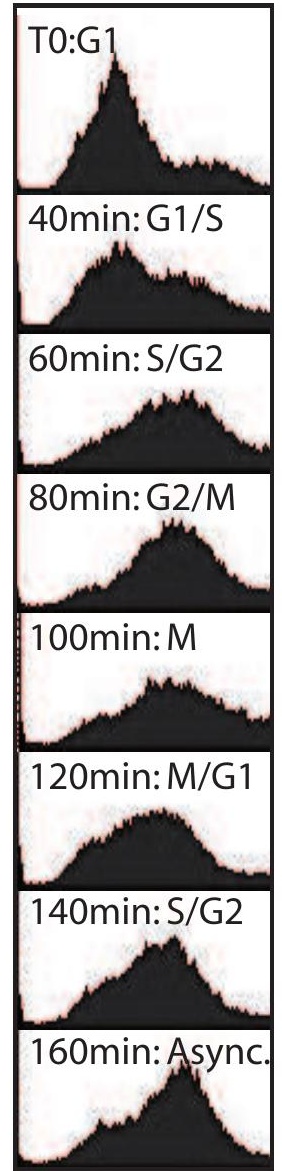

myc-Est2

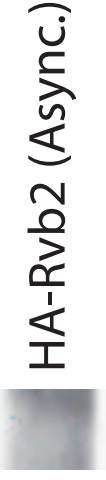

HA-Rvb2

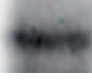

HA-Rvb2 myc-Est2 bar1

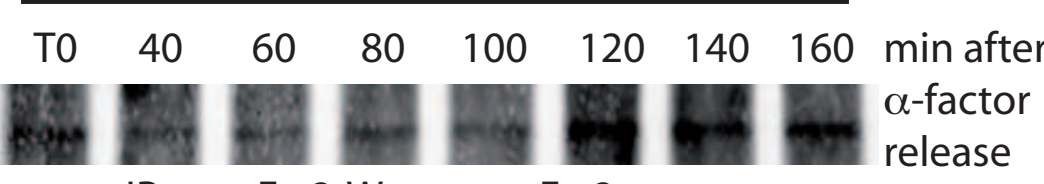

IP myc-Est2, West. myc-Est2

HA-Rvb2

$$
\text { IP HA-Rvb2, West. HA-Rvb2 }
$$



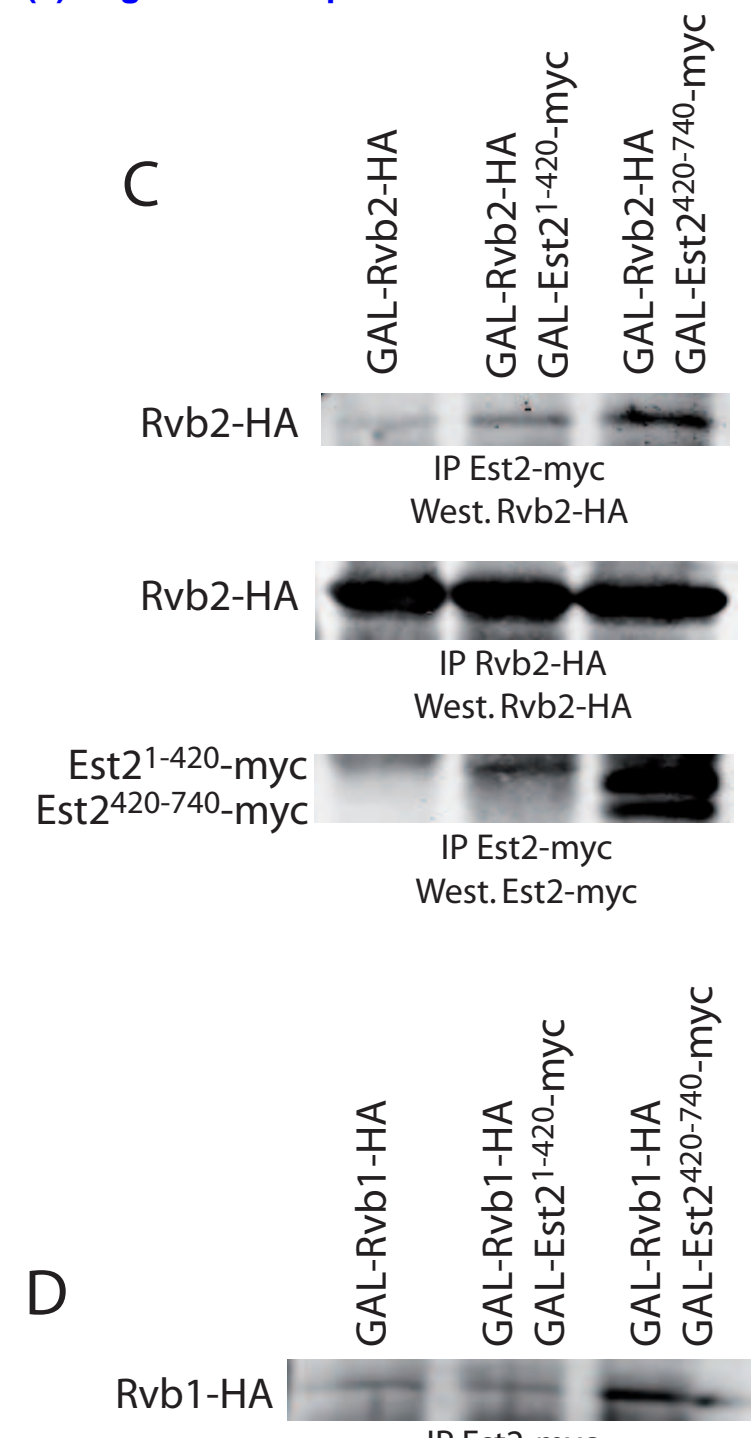

IP Est2-myc

West. Rvb1-HA

Rvb1-HA

IP Rvb1-HA

West. Rvb1-HA

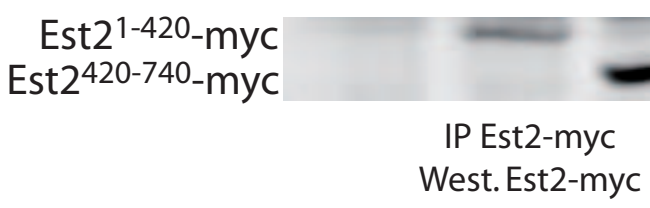

$\mathrm{E}$
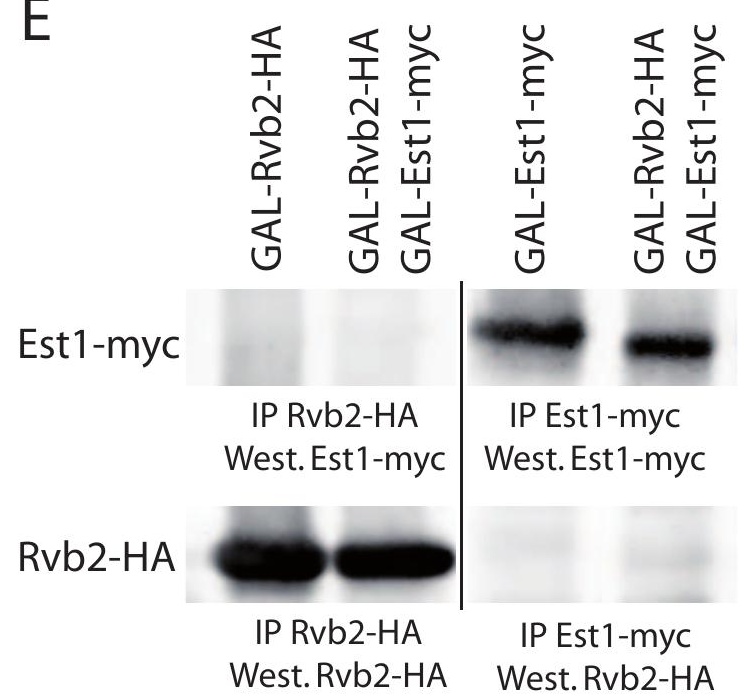


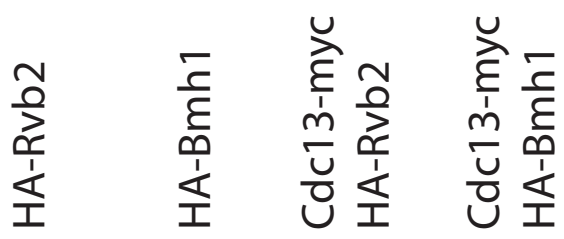

HA-Rvb2

HA-Bmh1

IP Cdc13-myc, West. HA-Rvb2/HA-Bmh1

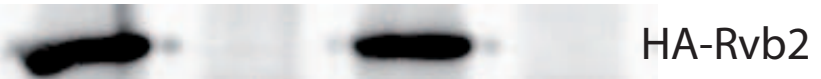

IP HA-Rvb2, West. HA-Rvb2

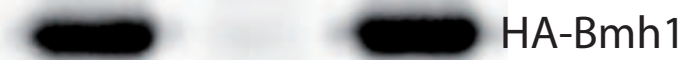

IP HA-Bmh1, West. HA-Bmh1

\section{I $=$ Cdc13-myc}

IP Cdc13-myc, West. Cdc13-myc

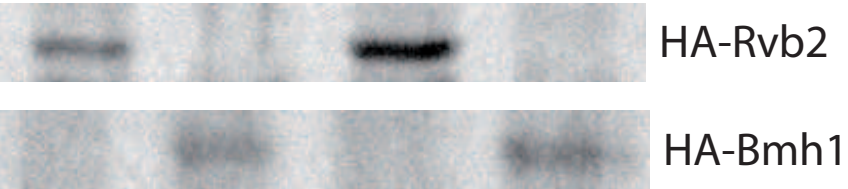

IP Cdc13-myc, West. HA-Rvb2/HA-Bmh1
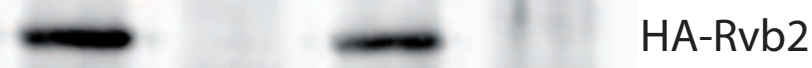

IP HA-Rvb2, West. HA-Rvb2

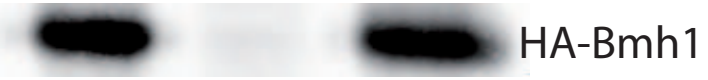

IP HA-Bmh1, West. HA-Bmh1

HA-Rvb2

\section{HA-Bmh1}

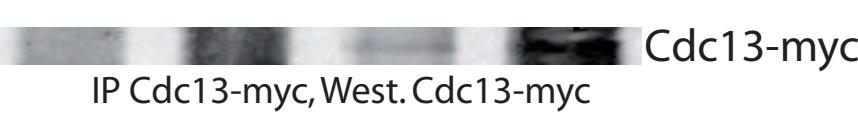
IP Cdc13-myc, West.Cdc13-myc Cdc13-myc
政

B
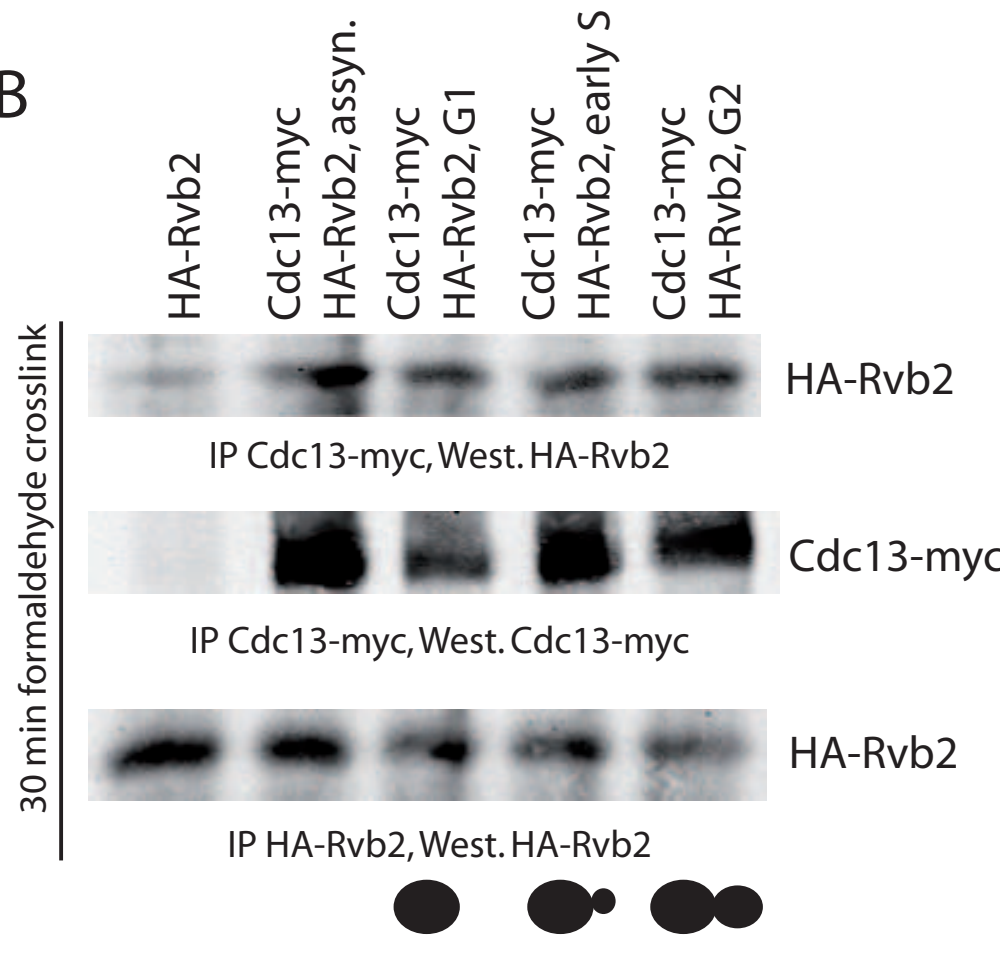

HA-Rvb2

IP Cdc13-myc, West. HA-Rvb2
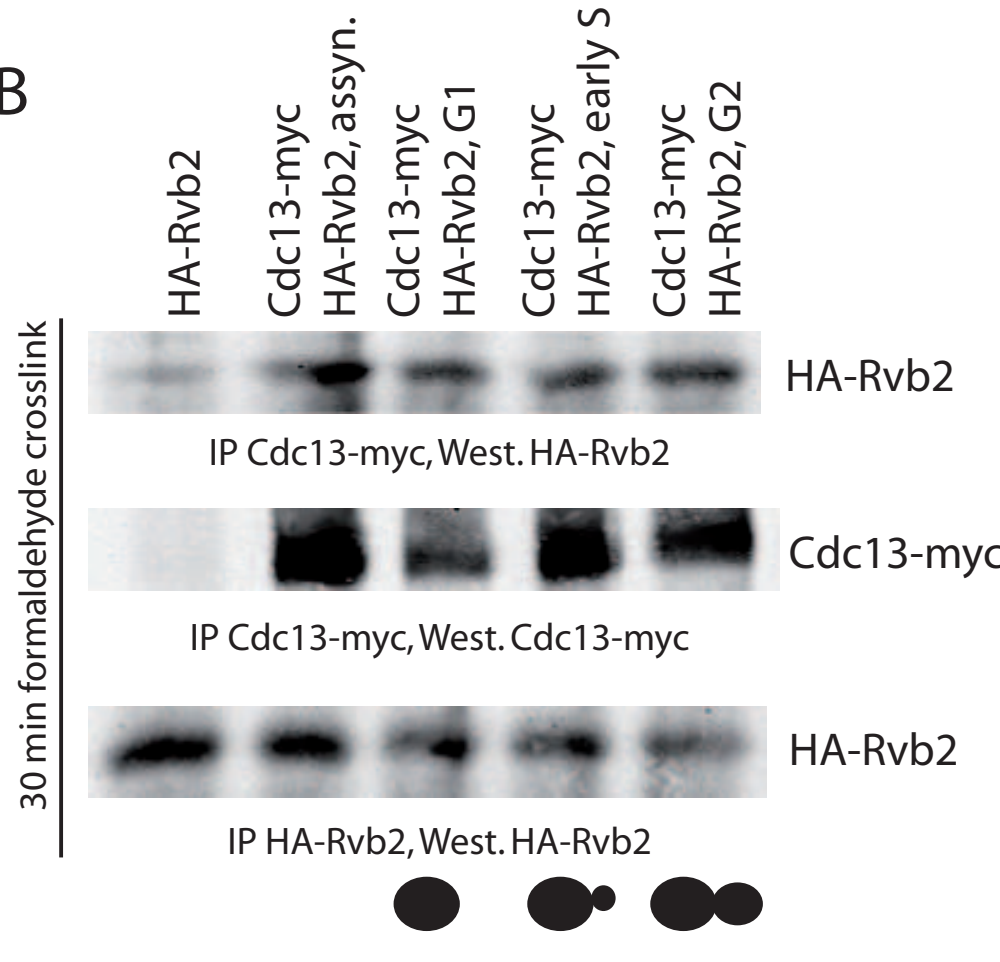

Cdc13-myc

IP Cdc13-myc, West. Cdc13-myc

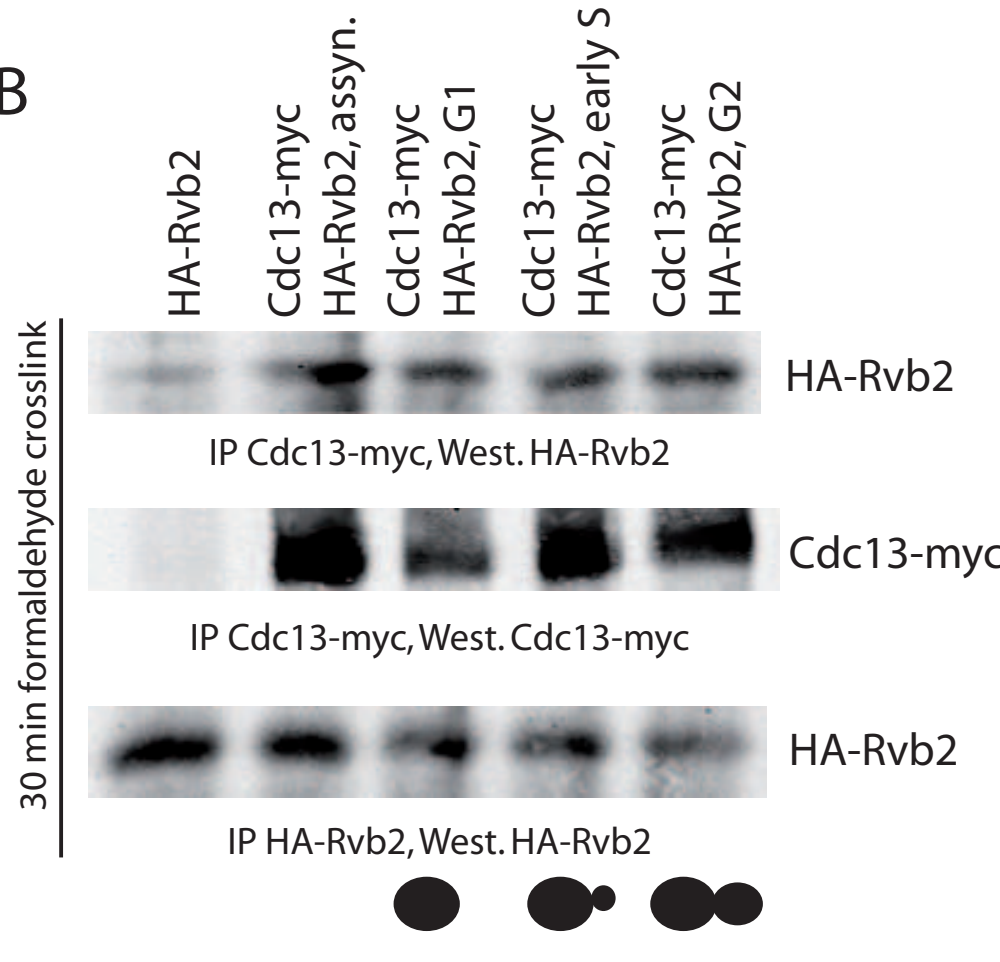

HA-Rvb2
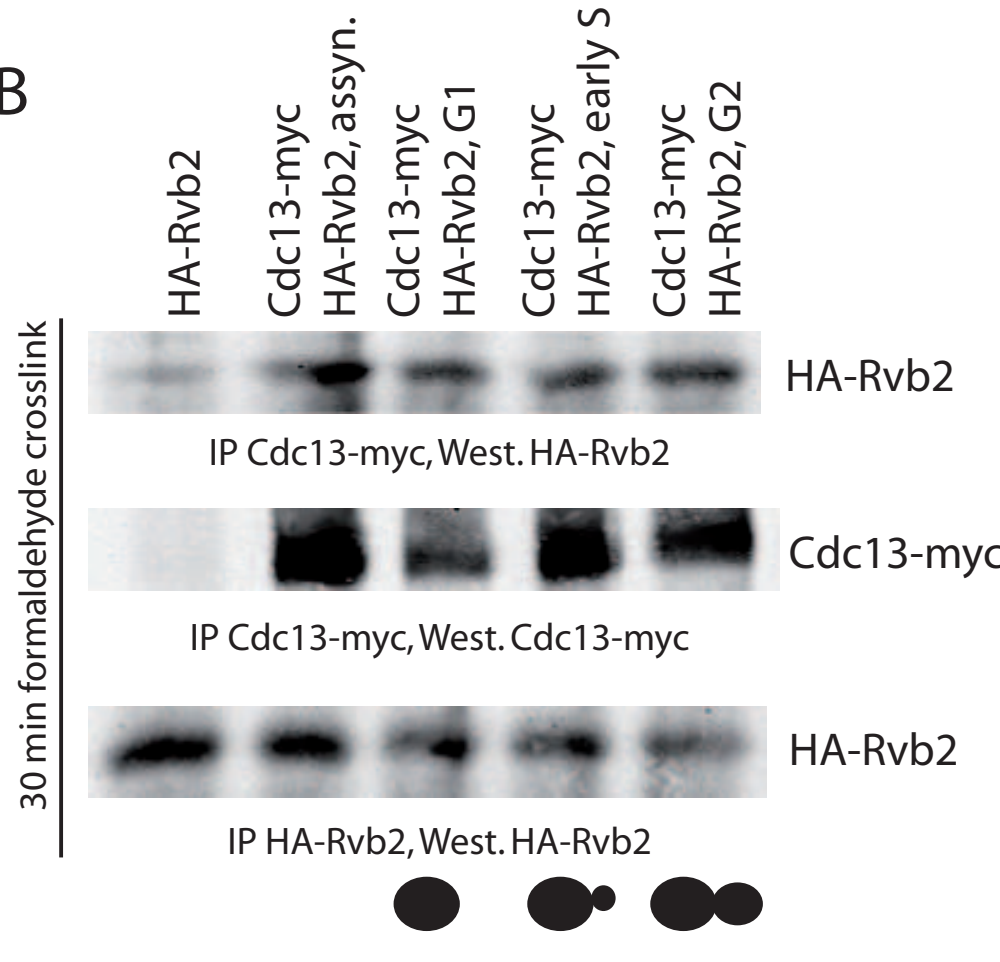

Pest. HA-Rvb2
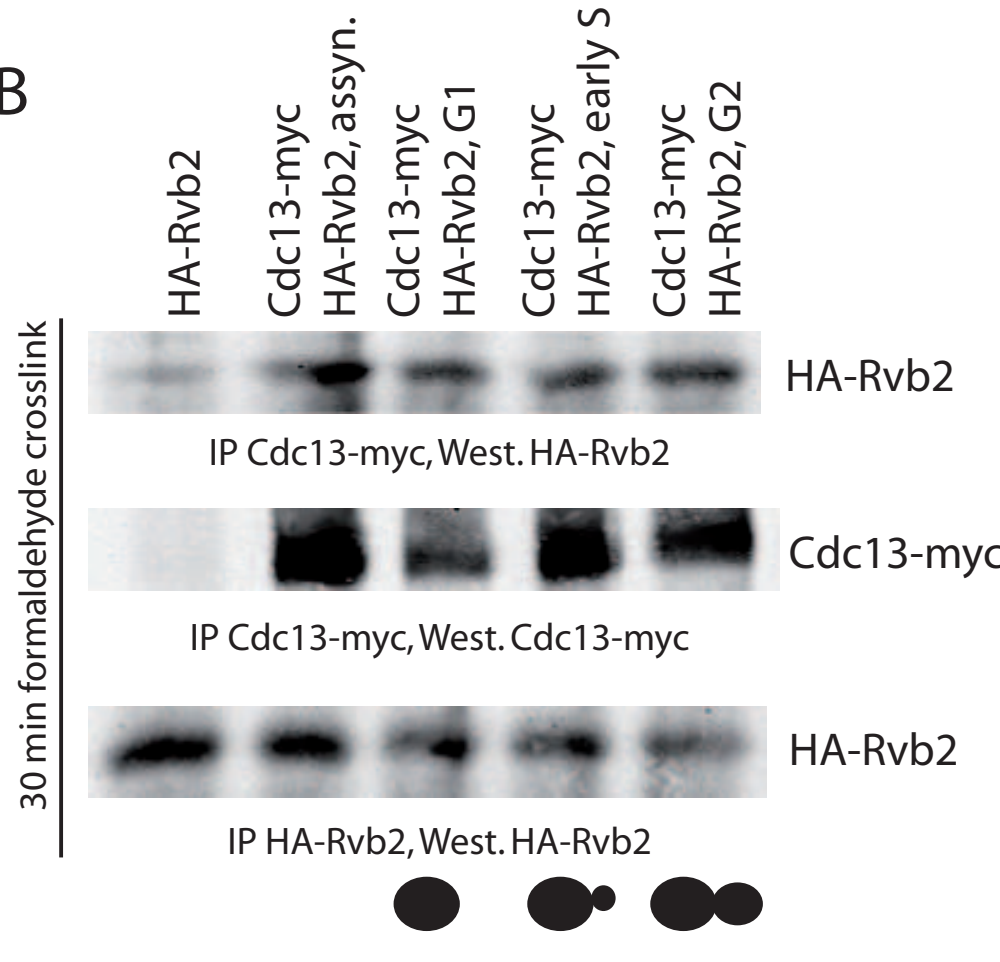

.

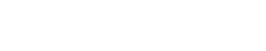

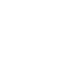


Figure 3

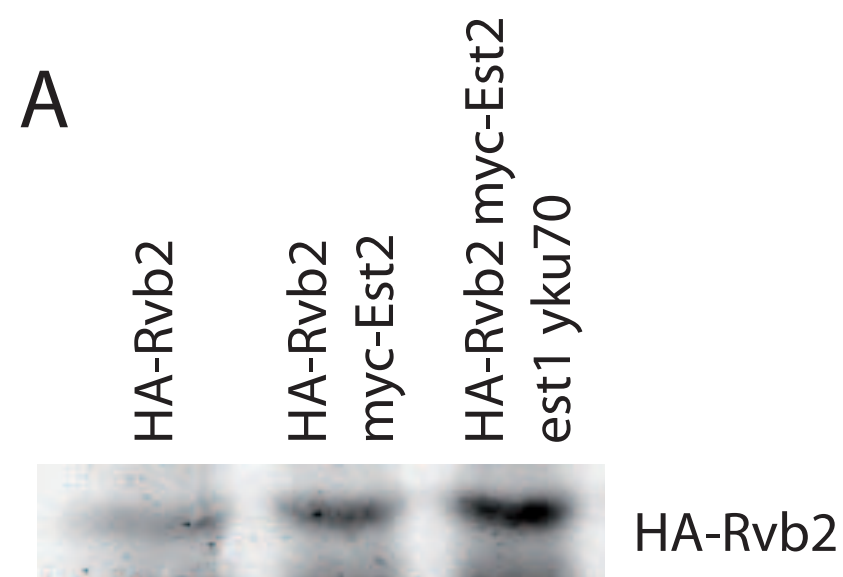

IP myc-Est2, West. HA-Rvb2

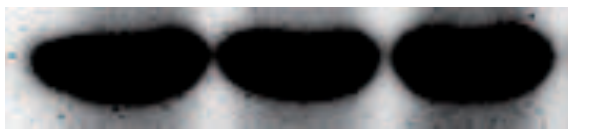

HA-Rvb2

IP HA-Rvb2, West. HA-Rvb2

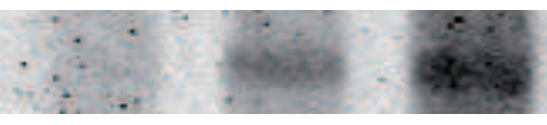

myc-Est2

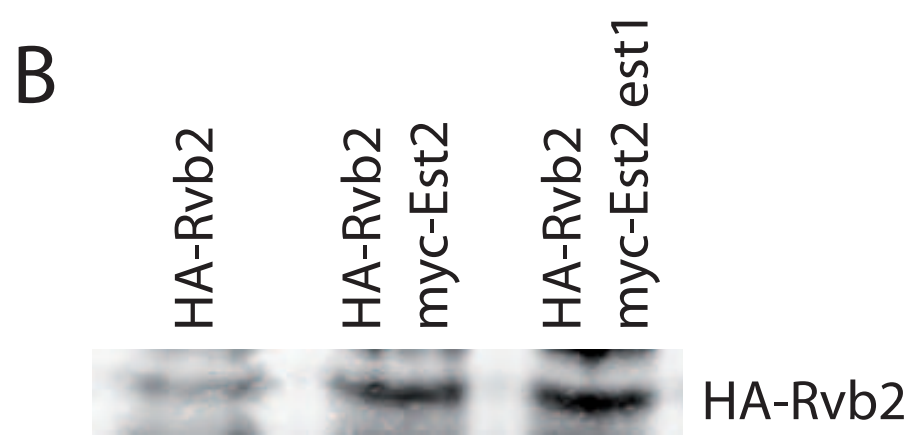

IP myc-Est2, West. HA-Rvb2

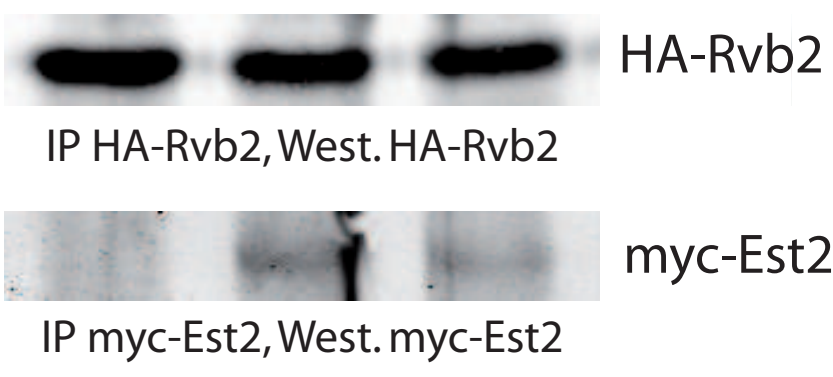


Click here to download Figure(s): Figure 4AB.eps

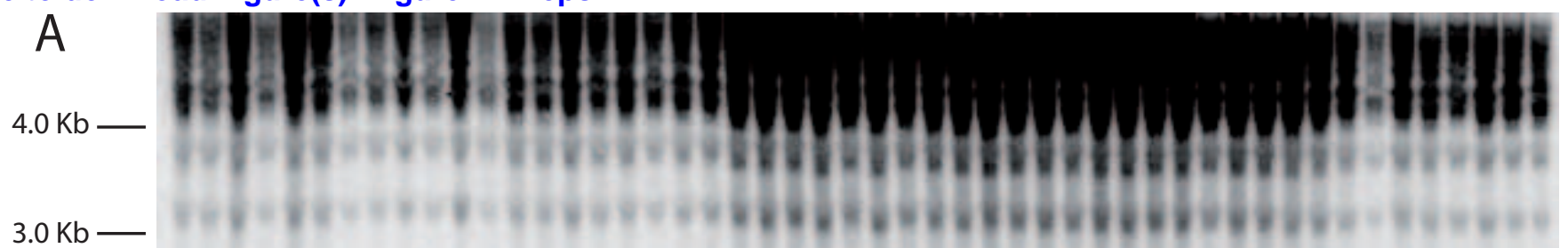

$.0 \mathrm{~Kb}-$

$1.6 \mathrm{~Kb}-1$

mean wild-type length

23

2426

wt
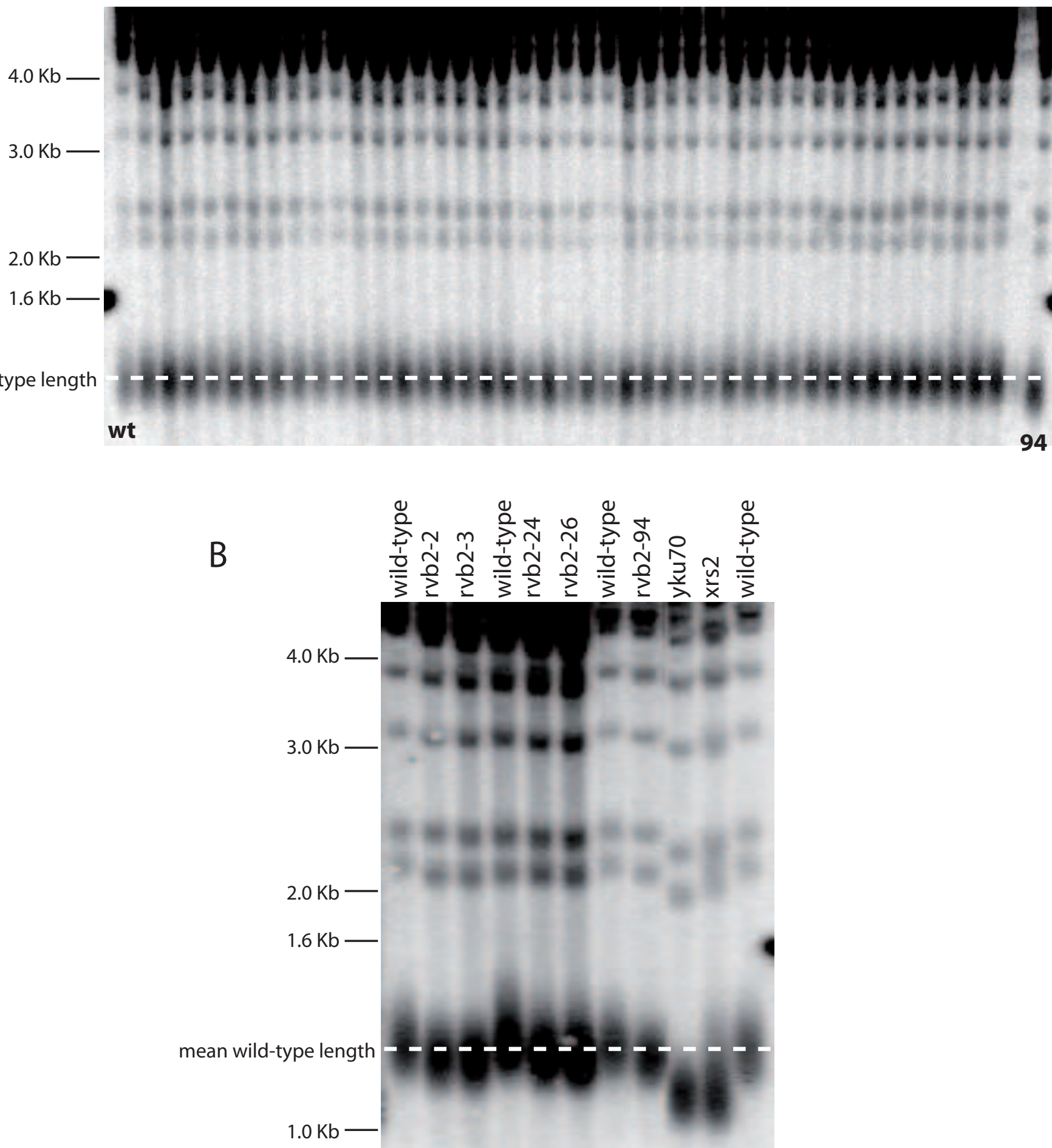

mean wild-type length 


\section{Figure $4 \mathrm{C}$}

$\mathrm{T}$

S. cerevisiae MSIQTSDPNETSDLKSLSLIAAHSHITGLGLDENLQPRPTSEGMVGQLQARRAAGVILKMVQNGTIAGRAVL $\mathrm{T}+\mathrm{E} \mathrm{D}++\mathrm{I}$ AHSHI GLGLD+ L+PR S+GMVGQL ARRAAGV+L+M++ G IAGRAVL H. sapiens MATVTATTKVPEIRDVTRIERIGAHSHIRGLGLDDALEPRQASQGMVGQLAARRAAGVVLEMIREGKIAGRAVL Walker A
73 VA GPPSTGKTA LAMGVSQSLGKDVPFTAIAGSEIFSLELSKTEALTQAFRKSIGIKIKEETELIEGEVVEIQIDRSITG-GH
+A P TGKTA+AMG++Q+LG D PFTAIAGSEIFSLE+SKTEALTQAFR+SIG++IKEETE+IEGEVVEIQIDR TG G
75 IAGQPGTGKTA AMMGMAQALGPDTPFTAIAGSEIFSLEMSKTEALTQAFRRSIGVRIKEETEIIEGEVVEIQIDRPATGTGS

155 KQGKLTIKTTDMETIYELGNKMIDGLTKEKVLAGDVISIDKASGKITKLGRSFARSRDYDAMGADTRFVQCPEGELQKRKTV K GKLT+KTT+METIY+LG KMI+ LTK+KV AGDVI+IDKA+GKI+KLGRSF R+RDYDAMG+ T+FVQCP+GELQKRK V 157 KVGKLTLKTTEMETIYDLGTKMIESLTKDKVQAGDVITIDKATGKISKLGRSFTRARDYDAMGSQTKFVQCPDGELQKRKEV

236 VHTVSLHEIDVINSRTQGFLALFTGDTGEIRSEVRDQINTKVAEWKEEGKAEIVPGVLFIDEVHMIDIECFSF INRALEDEF VHTVSLHEIDVINSRTQGFLALF+GDTGEI+SEVR+QIN KVAEW+EEGKAEI+PGVLFIDEVHMIDIE FSF+NRALE + 238 VHTVSLHEIDVINSRTQGFLALFSGDTGEIKSEVREQINAKVAEWREEGKAEI IPGVLFIDEVHMIDIESFSFLNRALESDM

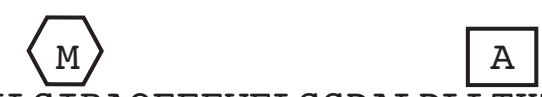

318 APIVMMATNRGVSKTRGTNYKSPHGLPLDLLDRSIIITTKSYNEQEIKTILSIRAQEEEVELSSDALDLLTKTGVETSLRYS AP+++MATNRG+++ RGT+Y+SPHG+P+DLLDR +I++T Y+E++ K IL IR +EE+VE+S DA +LT+ G+ETSLRY+ 320 APVLIMATNRGITRIRGTSYQSPHGIPIDLLDRLLIVSTTPYSEKDTKQILRIRCEEEDVEMSEDAYTVLTRIGLETSLRYA

400 SNLISVAQQIAMKRKNNTVEVEDVKRAYLLFLDSARSVKYVQENESQYIDDQGNVQISIAKSADPDAMDTTE $\mathrm{LI}+\mathrm{A}+\mathrm{KRK} \quad \mathrm{V}+\mathrm{V}+\mathrm{D}+\mathrm{KR} \mathrm{Y} \mathrm{LFLD}+\mathrm{RS}+\mathrm{Y}++\mathrm{E}+++$ 402 IQLITAASLVCRKRKGTEVQVDDIKRVYSLFLDESRSTQYMKEYQDAFLFNELKGETMDTS

$\operatorname{Rvb} 2-2, \operatorname{Rvb} 2-3 \bigcirc \operatorname{Rvb} 2-24, \operatorname{Rvb} 2-26$ $R v b 2-94$ 